\title{
One-year experience of oral substrate reduction therapy in three patients with Gaucher disease type I
}

\author{
Young Bae Sohn*๑, Yewon Kim ${ }^{\oplus}$, and Ji Eun Moon ${ }^{\oplus}$ \\ Department of Medical Genetics, Ajou University Hospital, Ajou University School of Medicine, Suwon, Korea
}

\begin{abstract}
Purpose: Eliglustat is an oral substrate reduction therapy (SRT) approved for adults with Gaucher disease type I (GD1) who are extensive, intermediate, or poor CYP2D6 metabolizers. Here we report one-year experience of eliglustat switch therapy from long-term enzyme replacement therapy (ERT) in three adult patients with GD1.

Materials and Methods: Medical history, clinical (hemoglobin concentration, platelet count, and bone mineral density) and biochemical parameters (angiotensin converting enzyme, total acid phosphatase, and lyso-gb1) of the patients were collected and evaluated by retrospective review of medical records at every 3, 6, or 12 month after switch to SRT.

Results: Patient 1 was a 43-year old female diagnosed GD1 and her clinical and biochemical parameters were stabilized for more than 20 years by ERT. Due to the burden of regular hospital visit, she switched to SRT. During one-year of SRT, clinical parameters and biomarkers were maintained stable. However, after suffering acute febrile illness during SRT, she decided to re-switch to ERT due to concerns about drug interaction. Patient 2 was 41-year old male, younger brother of patient 1 and Patient 3 was 31-year old male. They switched to SRT in clinically stable condition with long-term ERT. The one-year SRT was tolerable without specific safety issue and the clinical parameters were maintained stable.

Conclusion: One-year eliglustat therapy in three adult patients with GDI was generally tolerable and effective for maintaining the clinical parameters and biomarkers. However, the drug compliance, concurrent drug interactions, and long-term safety of eliglustat should be carefully monitored.
\end{abstract}

Key words: Gaucher disease, Drug therapy, Administration oral, Treatment outcome.

\section{Introduction}

Gaucher disease (OMIM 606463) is an inherited metabolic disorder caused by deficiency of the lysosomal enzyme acid $\beta$-glucocerebrosidase with consequential accumulation of the substrate, glucosylceramide, and its unacylated derivative, glucosylsphingosine, in lysosomes of tissue macrophages [1,2]. Clinical presentations include hepatosplenomegaly, anemia, thrombocytopenia, and bone disease [1]. Enzyme replacement therapy (ERT) has been a standard therapy of Gaucher disease for 25 years. However, life-long alternate-week infusion of deficient enzyme limits patients' quality of life. Eliglustat is an oral substrate reduction therapy (SRT) approved in the United States in 2014 and the European Union in 2015 for adults with Gaucher disease type I (GD1) who are extensive, intermediate, or poor CYP2D6 metabolizers [3-5]. Eliglustat partially inhibits the de novo biosynthesis of $\beta$-glucosylceramide, thereby rebalancing the rate of the substrate accumulation. The safety and efficacy

Received: 15 May 2020, Revised: 12 June 2020, Accepted: 12 June 2020, Published: 31 December 2020

${ }^{*}$ Corresponding author: Young Bae Sohn, M.D., Ph.D. (iD https://orcid.org/0000-0002-4664-1941

Department of Medical Genetics, Ajou University Hospital, Ajou University School of Medicine, 164 WorldCup-ro, Yeongtong-gu, Suwon 16499, Korea.

Tel: +82-31-219-4522, Fax: +82-31-219-4521, E-mail: ybsohn@ajou.ac.kr

Conflict of interest: The authors declare that they do not have any conflicts of interest.

(ㄷ) This is an open-access article distributed under the terms of the Creative Commons Attribution Non-Commercial License (http://creativecommons.org/licenses/by-nc/4.0/) which permits unrestricted non-commercial use, distribution, and reproduction in any medium, provided the original work is properly cited.

(c) Copyright 2020 by the Korean Society of Medical Genetics and Genomics 
of eliglustat therapy have been demonstrated in Phase 3 trials of previously untreated GD1 patients, as well as patients previously treated with ERT. In the phase 2 and 3 randomized, double-blind, placebo-controlled clinical trial, eliglustat therapy improved hematologic, visceral, and skeletal disease parameters relative to placebo in treatment-naïve patients after 9 to 12 months $[4,6]$, and these improvements continued with up to 8 years of eliglustat therapy in the open-label trial extension $[5,7]$. In the ENCORE trial of GD1 patients whose disease had been stable after a mean of 10 years of ERT, eliglustat was non-inferior to imiglucerase ERT [3], and the majority of patients maintained stable hematologic, visceral, and skeletal disease parameters for up to 4 years of eliglustat therapy [8].

In Korea, eliglustat was approved since 2018. Here we report two-year experience of eliglustat therapy in three adult patients with GD1 who were stabilized on over 20-year long-term ERT.

\section{Materials and Methods}

Three adult Korean patients (one female and two males) from two families of GD1 were enrolled this study. Medical history, clinical and biochemical parameters of the patients were collected and evaluated by retrospective review of medical records at every 3, 6, or 12 month after switch to SRT from ERT. The clinical parameters included hemoglobin concentration, platelet count, and bone mineral density (BMD) measured by dual-energy $x$-ray absorptiometry. Serum angiotensin converting enzyme, serum total acid phosphatase, and glucosylsphingosine (lyso-gb 1) concentration in dried blood spot were assessed as biomarker reflecting the activity of GD1. Adverse events were collected and assessed through regular follow-up in outpatient clinic every 1-2 months. The patients were educated for the drug interactions and avoidance of grapefruit during SRT. The drug adherence were monitored mainly by patients' self-report supported by clinical and biochemical parameters. This study was approved by the Institutional Review Board of the Ajou University Hospital (AJIRB-MED-18-364).

Table 1. Baseline clinical features of the patients

\begin{tabular}{|c|c|c|c|}
\hline & Patient 1 & Patient 2 & Patient 3 \\
\hline Sex/age (yr) & $\mathrm{F} / 43$ & $M / 41$ & $M / 31$ \\
\hline Age at diagnosis & 11 & 18 & 2 \\
\hline $\begin{array}{l}\beta \text {-glucocerebrosidase activity in } \\
\text { leukocyte (reference } \geq 8.7 \mathrm{nmoL} / \mathrm{hr} / \mathrm{mg} \text { prot) }\end{array}$ & 0.0 & 0.0 & 1.62 \\
\hline Genotype & p.[Gly46Glu];[Phe213lle] & p.[Gly46Glu];[Phe213lle] & p.[Gly46Glu];[Leu444Pro] \\
\hline History of surgery (age at surgery) & $\begin{array}{l}\text { Splenectomy (16) } \\
\text { Bilateral hip replacement (17) } \\
\text { Cesarean section (39) } \\
\text { Cholecystectomy (41) }\end{array}$ & None & Splenectomy (3) \\
\hline ERT duration before SRT (yr) & 22 & 21 & 22 \\
\hline Hematologic parameters & In normal range & In normal range & In normal range \\
\hline Skeletal involvement & $\begin{array}{l}\text { AVN of femoral heads } \\
\text { Osteopenia }\end{array}$ & Osteopenia & Osteopenia \\
\hline
\end{tabular}

F, female; M, male; ERT, enzyme replacement therapy; SRT, substrate reduction therapy; AVN, avascular necrosis.

(A)

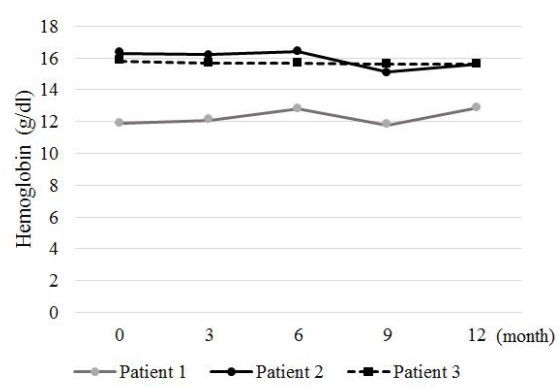

(B)

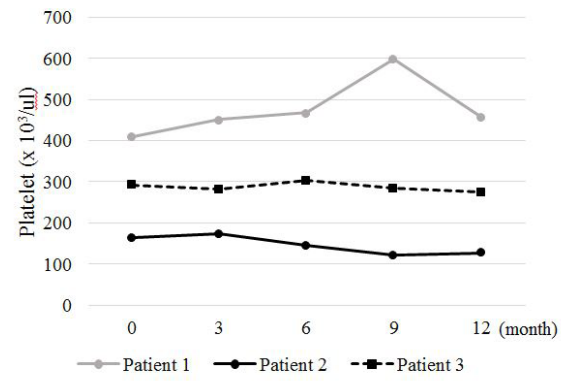

Fig. 1. Changes of hematologic parameters during one-year eliglustat therapy: hemoglobin concentration $(A)$ and platelet count (B). 


\section{Results}

The baseline clinical features are summarized in Table 1. Patient 1 and 2 were siblings diagnosed with GD 1 carrying the compound heterozygous mutation of $G B A$ (p.[Gly46Glu];:Phe213lle]). Their $\beta$-glucocerebrosidase activity in leukocyte was undetectable $(0 \mathrm{nmoL} / \mathrm{hr} / \mathrm{mg}$ prot, reference. 28.7). Patient 1 was 43 -year old female who were diagnosed GD1 due to anemia and thrombocytopenia with hepatosplenomegaly when she was 11 years old. At the time of diagnosis no specific therapy was available. She had splenectomy at 16 years old and got bilateral hip replacement surgery due to avascular necrosis of femoral heads at 17 years. Since ERT with imiglucerase was started when she was 21 years old, hematologic parameters and hepatomegaly have been normalized and stabilized for more than 20 years. During ERT, she was pregnant and successfully delivered a son by cesarean section at 39 years of age. And she got cholecystectomy due to acute cholecystitis at 40 years of age. Other long-term complications of GD including gaucheroma, monoclonal gammopathy have not occurred. Due to the burden of regular hospital visit for enzyme infusion and decreased compliance, the switch to the eliglustat therapy was started at 41 years old after evaluation of eligibility. She was extensive metabolizer determined by CYP2D6 genotyping. She had no concurrent medication. The dose of eliglustat was $84 \mathrm{mg}$ twice daily according to the drug label. At the beginning of the SRT, her hematologic parameters and liver size were in normal range. She had intermittent hip and knee pain which was tolerable in daily life. BMD revealed osteopenia of lumbar spines ( $T$ score was -1.0). Femoral BMD measurement was unavailable due to bilateral hip replacement surgery. She complained intermittent short palpitation resulting from premature ventricular contractions although routine electrocardiogram was normal. During eliglustat therapy, no serious adverse events were reported although mild headache and dyspepsia were noted. Her hematologic parameters (Fig. 1) and biomarkers (ACE and total acid phosphatase) were maintained stable except lyso-Gb1 (Fig. 2). Lumbar spinal BMD T score was increased to 0.1 from -1.0 after 12 months of eliglustat switch without any supplemental medication including calcium or vitamin D (Table 2). However, she had an acute febrile viral illness due to viral infection at 12 month after eliglustat therapy unfortunately. During the illness, she suffered nausea, abdominal discomfort, skin rashes that made her to skip eliglustat medication. The primary physicians
(A)

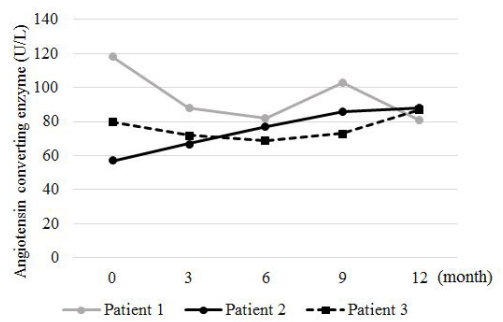

(C)

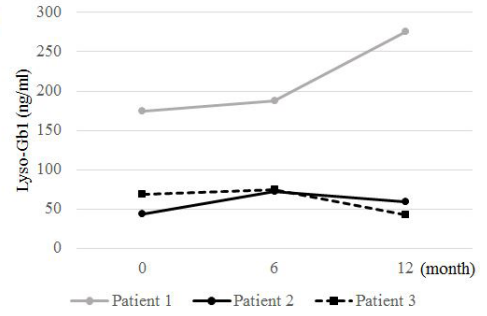

(B)

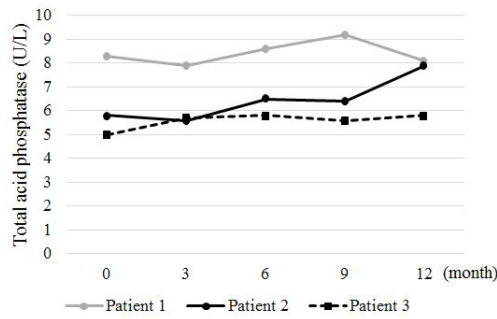

Table 2. Changes of BMD T score during one-year eliglustat therapy

\begin{tabular}{|c|c|c|c|c|c|c|}
\hline & \multicolumn{2}{|c|}{ Patient 1} & \multicolumn{2}{|c|}{ Patient 2} & \multicolumn{2}{|c|}{ Patient 3} \\
\hline & $0 \mathrm{~m}$ & $12 \mathrm{~m}$ & $0 \mathrm{~m}$ & $12 \mathrm{~m}$ & $0 \mathrm{~m}$ & $12 m$ \\
\hline Spine L1-L4 & -1.0 & 0.1 & -1.2 & -0.4 & -2.3 & -1.9 \\
\hline Femur neck & $N / A^{a}$ & $N / A^{a}$ & 0.5 & 0.3 & 0.3 & 0.5 \\
\hline Femur total & $N / A^{a}$ & $N / A^{a}$ & 0.9 & 0.9 & 0.8 & 1.2 \\
\hline
\end{tabular}

BMD, bone mineral density; N/A, not available.

afemoral BMD T scores were not available due to total hip replacement surgery in patient 1.

Fig. 2. Changes of biomarkers during oneyear eliglustat therapy: serum angiotensin converting enzyme (normal reference: 20 $70 \mathrm{U} / \mathrm{L})(\mathrm{A})$, serum total acid phosphatase (normal reference: 0.0-6.4 U/L) (B), and lyso-Gb1 in dried blood spot (normal reference: $<10 \mathrm{ng} / \mathrm{mL}$ ) (C). 
in local clinics refused to prescribe her medications for febrile illness because of concern for drug-drug interactions. The higher lyso-Gb1 concentration at 12 month after SRT seemed to be resulted from poor compliance during the acute febrile illness. Although she recovered completely from the acute illness, she decided to re-switch to ERT again after suffering this bad episode.

Patient 2 was 41 -year old male diagnosed GD1 at 18 years old by family screening. He had mild thrombocytopenia and splenomegaly at diagnosis. His genotype was same with patient 1. ERT with imiglucerase was started at 20 years of age and maintained his clinical and biochemical parameters stable for more than 20 years without any long-term complications of GD. He switched to eliglustat therapy at 40 years old after evaluation of eligibility (CYP2D6 genotype was extensive metabolizer). He had concurrent antihypertensive medication (losartan) which is not influence to the dose modification. He had $84 \mathrm{mg}$ twice daily eliglustat. The one-year eliglustat therapy was tolerable without specific adverse event. The hematologic parameters were maintained stable during eliglustat therapy (Fig. 1). Without any supplemental medication, Lumbar spinal BMD T score was increased to -0.4 from -1.2, femur BMD T score was maintained stable after 12 months of eliglustat switch (Table 2). However the biochemical parameters, especially the lyso-Gb1, was elevated after 6 month of therapy resulting from the decreased drug adherence. After re-education for the drug compliance, the drug compliance was enhanced and the lyso-Gb1 was decreased again (Fig 2C).

Patient 3 was 31-year old male diagnosed Gaucher disease at 2 years old by severe anemia, thrombocytopenia and hepatosplenomegaly. He had compound heterozygous mutations in GBA (p.[Gly46Glu];[Leu444Pro]) and the $\beta$-glucocerebrosidase activity in leukocyte was $1.62 \mathrm{nmoL} / \mathrm{hr} / \mathrm{mg}$ prot (reference $\geq 8.7$ ). He had splenectomy when he was 3 years old. In 1998, ERT was started at 9 years of age and his clinical and biochemical parameters maintained stable for more than 20 years without any long-term complications of GD. After graduating the university, he got job and the ERT compliance was decreased to once a month due to the difficulties of absence. He switched to eliglustat therapy at 30 years old after evaluation of eligibility (CYP2D6 genotype was extensive metabolizer). He had no concurrent medication during follow-up. The one-year eliglustat therapy was tolerable with stable hematologic parameters (Fig. 1) and biomarkers (Fig. 2). Lumbar spinal BMD T score was increased to -1.9 from -2.3 and femur BMD T score also slightly increased within normal range after 12 months of eliglustat switch (Table 2).

\section{Discussion}

In 2017, Cox et al. [8] demonstrated that eliglustat was noninferior to imiglucerase in maintaining the clinical parameters stable up to 4 years. Mean absolute values for hemoglobin concentration, platelet count, spleen volume, liver volume, and lumbar spine and femur $Z$ scores remained stable over 4 years. Biomarkers for disease activity were also found to be stable over time. Quality-of-life measures showed that the quality of life that they had achieved after a mean of 10 years on enzyme therapy was maintained while they were taking eliglustat therapy in the long term [8]. And, recent pooled analysis of longterm adverse event profile from four completed trials of eliglustat reported that the majority of the most frequently reported treatment-related adverse events were mild or moderate, transient, and occurred only once per patient [9].

Although the follow-up period was relatively short (12 months), this study also demonstrated that the hematologic parameters remained stable by switching to SRT in all three patients with GD1 who were stabilized with long-term ERT. One of the limitation of this study is that the quality-of-life measurement, the important parameter of SRT effectiveness, did not assessed. Notably, the lumbar spinal BMD T scores showed further improvement after SRT in all three patients although the femur BMD T scores were maintained stable (Table 2). It is uncertain whether the improvement of lumbar spinal BMD was resulted from SRT or not, because the follow-up period was too short to draw a confirmative conclusion. Further long-term follow-up data is needed with careful monitoring of BMD.

The 8-year long-term response of biomarkers (chitotriosidase, CCL18, lyso-Gb1, and Gb1) to eliglustat was consistent with clinical parameters, which were highly elevated in baseline, were reduced by $>85 \%$ [7]. Lyso-Gb1 is increasingly recognized as a highly specific and sensitive biomarker of GD with direct involvement in disease pathogenesis [10,11]. Despite the lysoGb1 level during ERT was unavailable in our patients, lyso-Gb1 remained stable during one-year of eliglustat therapy reflecting the stable disease activity. The significant reduction of lyso-Gb1 after initiation of ERT or SRT has been demonstrated, although the reduction becomes less robust over time after the maximal change rate [10-13]. Lyso-Gb1 was also useful for monitoring drug compliance during SRT. Recently, Hurvitz et al. [14] reported that lyso-Gb1 could reflect treatment response and detect treatment failures and compliance issues. In contrast to ERT, eliglustat is an oral drug and monitoring the drug compliance depends mainly on the patient's statements. Measuring lyso- 
Gb1 and sharing the result with the patient (especially patient 2 in this study) was effective for educating and emphasizing the drug adherence.

Because eliglustat is one of the newly introduced drug, concerns about drug-drug or drug-food interactions were arose not only from the patients but also the physicians in real-world setting. The patient 1 decided to return to ERT because of uncertain concerns about possible drug interactions after suffering acute illness needs concurrent medication. As with many other oral drugs, eliglustat is metabolized primarily through the CYP2D6 pathway [15]. Dosing in the drug label is based on the CYP2D6 genotype. Caution is required when patients are taking other CYP2D6 and CYP3A4 inhibitors because this may increase the availability of eliglustat and worsen adverse effects [15]. For example, the patients should avoid grapefruit juice because it is a strong CYP3A4 inhibitor [15]. The label also makes recommendations with regard to concomitant medications that might increase or decrease the plasma drug levels $[16,17]$. Physicians need caution for prescribing drugs for concomitant chronic condition or acute illness. And patients should be educated for need of dose-adjustment with concomitant medicine.

This study described the one-year experience of eliglustat therapy in three adult patients with GD1 who were clinically stabilized by over 20-year ERT. Although more long-term data is needed, eliglustat therapy was generally tolerated and the clinical parameters were remained stable. Considering the merits of oral drug, eliglustat could be one of the treatment option in eligible patients with GD1. Furthermore, education and caution for drug interaction and compliance is needed not only for patients but also physician for successful switching and achieving treatment goals.

\section{References}

1. Grabowski GA, Petsko GA, Kolodny EH. Gaucher disease. In: Valle DL, Antonarakis S, Ballabio A, Beaudet AL, Mitchell GA, eds. The online metabolic and molecular bases of inherited disease. New York: McGraw-Hill, 2019.

2. Cox TM, Cachón-González MB. The cellular pathology of lysosomal diseases. J Pathol 2012;226:241-54.

3. Cox TM, Drelichman G, Cravo R, Balwani M, Burrow TA, Martins $A M$, et al. Eliglustat compared with imiglucerase in patients with Gaucher's disease type 1 stabilised on enzyme replacement therapy: a phase 3 , randomised, open-label, non-inferiority trial. Lancet 2015;385:2355-62.

4. Mistry PK, Lukina $E_{1}$ Ben Turkia $H_{1}$ Amato $D$, Baris $H$, Dasouki $M$, et al. Effect of oral eliglustat on splenomegaly in patients with Gaucher disease type 1: the ENGAGE randomized clinical trial. JAMA 2015;313:695-706.

5. Mistry PK, Lukina E, Ben Turkia H, Shankar SP, Baris H, Ghosn M, et al. Outcomes after 18 months of eliglustat therapy in treatment-naïve adults with Gaucher disease type 1: the phase 3 ENGAGE trial. Am J Hematol 2017;92:1170-6.

6. Lukina E, Watman N, Arreguin EA, Banikazemi M, Dragosky M, lastrebner $M$, et al. A phase 2 study of eliglustat tartrate (Genz-112638), an oral substrate reduction therapy for Gaucher disease type 1. Blood 2010;116:893-9.

7. Lukina E, Watman N, Dragosky M, Lau H, Avila Arreguin E, Rosen-

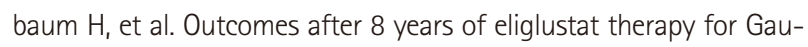
cher disease type 1: final results from the phase 2 trial. Am J Hematol 2019;94:29-38.

8. Cox TM, Drelichman G, Cravo R, Balwani M, Burrow TA, Martins $A M$, et al. Eliglustat maintains long-term clinical stability in patients with Gaucher disease type 1 stabilized on enzyme therapy. Blood 2017;129:2375-83.

9. Peterschmitt MJ, Freisens S, Underhill LH, Foster MC, Lewis G, Gaemers SJM. Long-term adverse event profile from four completed trials of oral eliglustat in adults with Gaucher disease type 1. Orphanet J Rare Dis 2019;14:128.

10. Dekker N, van Dussen L, Hollak CE, Overkleeft H, Scheij S, Ghauharali $\mathrm{K}$, et al. Elevated plasma glucosylsphingosine in Gaucher disease: relation to phenotype, storage cell markers, and therapeutic response. Blood 2011;118:e118-27.

11. Murugesan V, Chuang WL, Liu J, Lischuk A, Kacena K, Lin H, et al. Glucosylsphingosine is a key biomarker of Gaucher disease. Am J Hematol 2016;91:1082-9.

12. Rolfs A, Giese AK, Grittner U, Mascher D, Elstein D, Zimran A, et al. Glucosylsphingosine is a highly sensitive and specific biomarker for primary diagnostic and follow-up monitoring in Gaucher disease in a non-Jewish, Caucasian cohort of Gaucher disease patients. PLoS One 2013:8:e79732.

13. Elstein D, Mellgard B, Dinh Q, Lan L, Qiu Y, Cozma C, et al. Reductions in glucosylsphingosine (lyso-Gb1) in treatment-naïve and previously treated patients receiving velaglucerase alfa for type 1 Gaucher disease: data from phase 3 clinical trials. Mol Genet Metab 2017; 122:113-20.

14. Hurvitz N, Dinur T, Becker-Cohen M, Cozma C, Hovakimyan M, Op-

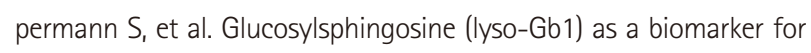
monitoring treated and untreated children with Gaucher disease. Int J Mol Sci 2019;20:3033.

15. Bennett LL, Turcotte K. Eliglustat tartrate for the treatment of adults with type 1 Gaucher disease. Drug Des Devel Ther 2015;9:4639-47. 
16. Balwani M, Burrow TA, Charrow J, Goker-Alpan O, Kaplan P, Kishnani PS, et al. Recommendations for the use of eliglustat in the treatment of adults with Gaucher disease type 1 in the United States. Mol Genet Metab 2016;117:95-103.
17. Belmatoug $N$, Di Rocco $M$, Fraga $C$, Giraldo $P$, Hughes $D$, Lukina $E$, et al. Management and monitoring recommendations for the use of eliglustat in adults with type 1 Gaucher disease in Europe. Eur J Intern Med 2017;37:25-32. 